\title{
Stochastic multi-depot capacitated vehicle routing problem with pickup and delivery: heuristic approaches
}

\author{
Brenner H. O. Rios $^{1}$, Eduardo C. Xavier ${ }^{1}$, Flávio K. Miyazawa ${ }^{1}$, Pedro Amorim ${ }^{2}$ \\ ${ }^{1}$ Institute of Computing - University of Campinas (UNICAMP) \\ Campinas - SP - Brazil \\ ${ }^{2}$ Faculty of Engineering - University of Porto \\ Porto, Portugal. \\ brenner@students.ic.unicamp.br, \{ecx, fkm\}@ic.unicamp.br, \\ amorim.pedro@fe.up.pt
}

\begin{abstract}
We present a natural probabilistic variation of the multi-depot vehicle routing problem with pickup and delivery. We denote this variation by Stochastic multi-depot capacitated vehicle routing problem with pickup and delivery (SMCVRPPD). We present an algorithm to compute the expected length of an a priori route under general probabilistic assumptions. To solve the SMCVRPPD we propose an Iterated Local Search (ILS) and a Variable Neighborhood Search (VNS). We evaluate the performance of these heuristics on a data set adapted from TSPLIB instances. The results show that the ILS is effective to solve SMCVRPPD.
\end{abstract}

\section{Introduction}

After several decades of development, the vehicle routing problem (VRP) has become an important content in logistics management research, and has been widely used in transportation system, logistics distribution system and express delivery system [Cao and Yang 2017]. Several versions of the vehicle routing problem have been proposed, such as the capacitated vehicle routing problem (CVRP), multi-depot vehicle routing problem (MDVRP) and vehicle routing problem with pickups and deliveries (VRPPD) [Oyola et al. 2017]. To better describe the real world, stochastic data may be included in the model. In this case a new problem arises, stochastic multi-depot capacitated vehicle routing problem with pickup and delivery (SMCVRPPD). We provide a description of the SMCVRPPD in Section 2. Section 3 presents the an algorithm to compute the expected length of an a priori route. Section 4 describes the proposed heuristic and computational experiments. Section 5 presents the conclusions of this work.

\section{Problem description}

In this work, SMCVRPPD is defined as follows. Let $G=(V, E)$ be a complete undirected graph, where $V=\left\{v_{1}, \ldots, v_{n}\right\}$ is the vertex set and $E=\left\{\left(v_{i}, v_{j}\right): v_{i}, v_{j} \in V, i<j\right\}$ is the edge set. With each edge $\left(v_{i}, v_{j}\right)$, it is associated a non-negative cost or distance $d_{i j}$. A subset of vertices $D=\left\{v_{1}, \ldots, v_{m}\right\}$ represents the depots, and the remaining vertices $V^{\prime}=\left\{v_{m+1}, \ldots, v_{n}\right\}$ corresponds to pickup and delivery points. Let $w=\left|V^{\prime}\right| / 2$, then $w$ vertices are pickup points and $w$ vertices are delivery points. Each pickup point $v_{i}$ is associated with a unique delivery point $v_{i+w}$, and vice versa, for $m+1 \leq i \leq m+w$. 
There are $m$ identical vehicles of unlimited capacity such that each one is located in a single depot. Each vehicle leaves its depot, serves a subset of pickup and delivery vertices and returns to its depot, forming a cycle (or route). The problem consists in determining a set of $m$ vehicle cycles of minimal total cost considering the following constraints: a) each cycle starts and ends at the corresponding vehicles depot; b) each $v \in V^{\prime}$ is visited exactly once by one vehicle c) each pair of pickup and delivery points, e.g $\left\{v_{i}, v_{i+w}\right\}$ for $m+1 \leq i \leq m+w$, must belong to the same cycle and d) each cycle has an orientation where each pickup vertex in this cycle appears before its delivery pair.

The SMCVRPPD has one type of uncertainty: stochastic pickup and delivery points. Each subset $\left\{v_{i}, v_{i+k}\right\} \in V^{\prime}$, for $m+1 \leq i \leq m+k$, has a probability $p_{i}$ of being present. When pickup point $v_{i}$ is absent, delivery point $v_{i+k}$ is also absent, and its demand $\xi_{i}$ is equal to zero otherwise is equal to 1 . In this work, we assume all demands are unitary (1 or 0$)$, discrete and independent. In a first stage, a set of cycles satisfying conditions a)- d) of the SMCVRPPD are determined. The presence or absence of $\left\{v_{i}, v_{i+k}\right\}$ is revealed at the latest upon leaving the preceding vertex of $v_{i}$, but the positive demand of every remaining customer becomes known only when the vehicle arrives at the delivery's location. In a second stage, the first stage routes are followed as planned, with the following exceptions: 1) any absent customer is skipped and 2) whenever the remaining vehicle capacity is not enough to carry current node demand, the vehicle follows a strategy, in either case, route failure is said to occur and a strategy used to deal with the problem failure. We use the following strategy: once the vehicle reaches its maximum capacity, a penalty is paid for the transportation of excess cargo by an outsourced service, and the vehicle continues the original route. The SMCVRPPD consists of designing a first stage solution to minimize the expected cost of the second stage solution.

\section{The expected cost of an a priori route}

Given a priori computed route $s=\left(v_{0}, v_{1}, \ldots, v_{2 q}, v_{0}\right)$, where $v_{0}$ is a depot, let $l_{s}$ be the cost/length of $s$. Our goal is to compute the expected length $E\left[l_{s}\right]$ of route $s$, given that during its execution, each pair $\left\{v_{i}, v_{i+w}\right\}$ of pickup and delivery points in this route have a probability of occurring during $s$ 's execution. We may also refer to node $v_{i}$ as $r_{i}$, and $v_{i+w}$ as $c_{i}$. Let $P\left(v_{i}\right)$ be the probability that node $v_{i}$ appears in $s$, therefore $P\left(r_{i}\right)=P\left(c_{i}\right)$. In this work we use the following strategy, each time the maximum capacity $Q$ of the vehicle is reached, a penalty must be paid. Such penalty represents the cost of travel from the pickup point where the vehicle was full to its associated delivery point. Then the vehicle load is updated to $Q-1$ and the vehicle continues the original route. To compute the

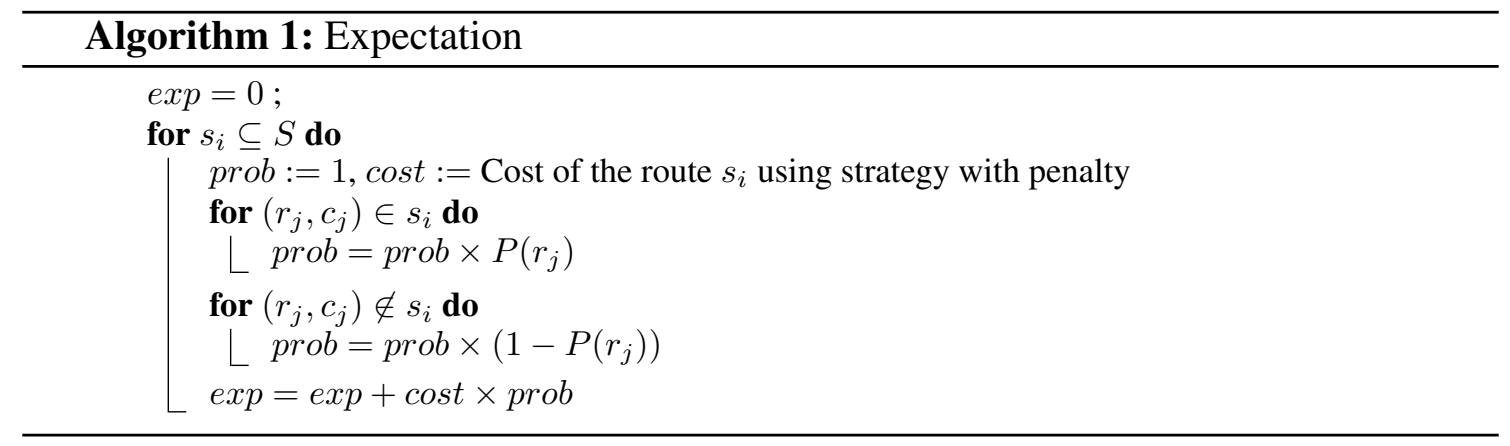


expected cost $E\left[l_{s}\right]$ of a route $s$ we use Algorithm 1 . Let $s_{i}$ be a valid route generated from the route $s$, e.g., $s_{1}=\left(v_{0}\right)$ and $s_{2}=\left(v_{0}, r_{1}, c_{1}, v_{0}\right)$ are valid routes. In $s_{1}$ no pair of pickup and delivery appear in the route. In $s_{2}$ only the pair $\left(r_{1}, c_{1}\right)$ appears. $S$ denotes the set of valid routes generated from $s$. There are $2^{q}$ valid routes generated from $s$.

\section{Heuristics approaches}

We present the local search operators, perturbation operators, and heuristics used by VNS and ILS. We adapt the algorithms VNS [Rios et al. 2020b] and ILS [Rios et al. 2020a] for the SMCVRPPD. This means that the operators are executed following the sequence of the original algorithms.

\subsection{Initial solution generation}

The method employed for building a feasible initial solution is based in the work of [Kuo and Wang 2012], so it is generated by following two steps. The first step is called nodes assignment. Each pair of pickup and delivery is assignment to one of the depots. After all vertices have been assigned to depots, the second step, called nodes sequencing, decides the service sequence of the pickup and delivery nodes.

\subsection{Local search}

A set $\left\{N^{1}, \ldots, N^{6}\right\}$ of six neighborhood operators were used by the proposed heuristics. All operators are exhaustively executed. These operators are adapted in such a way that they preserve feasibility. The list of neighborhoods considered are:

- Shift(1,0) - $N^{1}$ - A pickup and delivery pair $r, c$ is removed from a route $t_{1}$ and each one is moved to the best position in route $t_{2}$ keeping the feasibility of the solution.

- $\operatorname{Swap}(1,1)-N^{2}-$ An exchange between a pair $r_{1}, v_{1}$ from a route $t_{1}$ and another pair $r_{2}, v_{2}$ from route $t_{2}$. Each vertex of the pairs are inserted in the best possible position while maintaining the feasibility of the solution.

- Mix-Shift(1,0) - $N^{3}$ - This operator is similar to the $\operatorname{Shift}(1,0)$ operator with the difference that now it is allowed a movement within its own route.

- 2-opt - $N^{4}$ - Two nonadjacent arcs are removed and other two are added to form a new route. We only consider movements that do not break the constraints of the problem.

- 3-opt $-N^{5}$ - Three nonadjacent arcs are removed and other two are added to form a new route. We only consider movements that do not break the constraints of the problem.

- Reverse $-N^{6}$ - This operator reverses the direction of the route. Then swaps are performed between each pair of pickup and delivery.

\subsection{Perturbation Mechanism}

A set $P$ of two perturbation mechanisms were adopted in the heuristics. Every time the perturb() function is called one of the following operators is randomly selected and applied.

- Double-Swap - $P^{1}-$ Two $\operatorname{Swap}(1,1)$ operators are performed in sequence.

- Depot Exchange - $P^{2}$ - The depot exchange operator select two depots at random, and exchange their routes. 


\begin{tabular}{|c|c|c|c|c|c|c|c|c|c|}
\hline \multicolumn{4}{|c|}{ Instance } & \multicolumn{3}{|c|}{ ILS } & \multicolumn{3}{|c|}{ VNS } \\
\hline Name & $|V|$ & $D$ & $Q$ & Best Cost & Avg. Cost & Time (s) & Best Cost & Avg. Cost & Time (s) \\
\hline bayg29a & 29 & 3 & 4 & 378.60 & 652.96 & 23.51 & 378.60 & 670.93 & 25.10 \\
\hline bayg $29 b$ & 29 & 9 & 4 & 3185.55 & 4388.36 & 10.77 & 4055.68 & 4661.49 & 12.38 \\
\hline bayg29c & 29 & 9 & 2 & 1717.82 & 2134.55 & 3.84 & 1836.92 & 2161.74 & 4.14 \\
\hline dantzig42a & 42 & 8 & 4 & 272.98 & 348.09 & 3.26 & 288.99 & 350.88 & 3.55 \\
\hline dantzig $42 b$ & 42 & 4 & 4 & 91.24 & 141.94 & 1539.08 & 107.75 & 144.41 & 1558.08 \\
\hline dantzig42c & 42 & 10 & 2 & 304.77 & 413.34 & 2.55 & 354.52 & 419.87 & 2.85 \\
\hline eil51a & 51 & 3 & 4 & 60.41 & 117.29 & 8282.83 & 106.39 & 131.08 & 8382.83 \\
\hline eil51b & 51 & 7 & 5 & 79.67 & 112.04 & 123.72 & 86.43 & 112.71 & 134.72 \\
\hline eil51c & 51 & 9 & 3 & 136.15 & 193.84 & 8.34 & 159.49 & 196.17 & 8.45 \\
\hline
\end{tabular}

\section{Computational experiments}

We conducted experiments using a data set derived from three TSPLIB instances ( bayg29, dantzig42 and eil51). For each of these instances, $n$ vertices in the interval of $[2,10]$ were randomly selected to be depots. The capacity of each vehicle $Q$ was randomly generated in the interval $[2,10]$. A random matching was performed among the other vertices to create pickup and delivery pairs. The probability of presence of each pickup and delivery pair was chosen uniformly in the interval $[0,1]$. We generate 9 test instances. The proposed heuristic (VNS and ILS) were coded in C++ and all experiments were run on a Linux operating system with 3 GB memory and Intel Core i5 2.54x $4 \mathrm{Ghz}$ processor. Computational times reported here are in CPU seconds on this machine. Ten independent runs of the algorithms were performed for each test case.

The results show that the ILS was superior for all instances tested. Our approach can be used as benchmark for future research in this area. The SMCVRPPD can be further generalized to handle more practical constraints, e.g., time windows and stochastic demands.

\section{References}

Cao, W. and Yang, W. (2017). A survey of vehicle routing problem. In MATEC Web of Conferences, volume 100, page 01006. EDP Sciences.

Kuo, Y. and Wang, C.-C. (2012). A variable neighborhood search for the multi-depot vehicle routing problem with loading cost. Expert Systems with Applications, 39(8):6949_ 6954.

Oyola, J., Arntzen, H., and Woodruff, D. L. (2017). The stochastic vehicle routing problem, a literature review, part ii: solution methods. EURO Journal on Transportation and Logistics, 6(4):349-388.

Rios, B., Xavier, E. C., Miyazawa, F. K., and Amorim, P. (2020a). Multi-depot multiple tsp with pickup and delivery: an vns approach. In ANAIS DO LII SIMPóSIO BRASILEIRO DE PESQUISA OPERACIONAL (SBPO).

Rios, B., Xavier, E. C., Miyazawa, F. K., and Amorim, P. (2020b). Stochastic multidepot vehicle routing problem with pickup and delivery: an ils approach. In 2020 15th Conference on Computer Science and Information Systems (FedCSIS), pages 307-315. IEEE. 